\title{
Chemical evolution of the Galaxy disk in connection with large-scale winds
}

\author{
Takuji Tsujimoto $^{1}$, Joss Bland-Hawthorn ${ }^{2}$, and Kenneth C. Freeman ${ }^{3}$ \\ ${ }^{1}$ National Astronomical Observatory, Mitaka-shi, Tokyo 181-8588, Japan \\ email: taku.tsujimoto@nao.ac.jp \\ ${ }^{2}$ Institute of Astronomy, School of Physics, University of Sydney, NSW 2006, Australia \\ ${ }^{3}$ Research School of Astronomy and Astrophysics (RSAA), Australian National University, \\ Cotter Road, Weston Creek, ACT 2611, Australia
}

\begin{abstract}
Comparison of elemental abundance features between old and young thin disk stars may reveal the action of ravaging winds from the Galactic bulge, which once enriched the whole disk, and set up the steep abundance gradient in the inner disk $\left(R_{\mathrm{GC}} \lesssim 10-12 \mathrm{kpc}\right)$ and simultaneously the metallicity floor $([\mathrm{Fe} / \mathrm{H}] \sim-0.5)$ in the outer disk. After the end of a crucial influence by winds, chemical enrichment through accretion of a metal-poor material from the halo onto the disk gradually reduced the metallicity of the inner region, whereas an increase in the metallicity proceeded beyond a solar circle. This results in a flattening of abundance gradient in the inner disk, and our chemical evolution models confirm this mechanism for a flattening, which is in good agreement with the observations. Our scenario also naturally explains an observed break in the metallicity floor of the outer disk by young stars since the limit of self-enrichment in the outer disk is supposed to be $[\mathrm{Fe} / \mathrm{H}] \lesssim-1$ and inevitably incurs a direct influence of the dilution by a low-metal infall whose metallicity is $[\mathrm{Fe} / \mathrm{H}] \sim-1$. Accordingly, we propose that the enrichment by large-scale winds is a crucial factor for chemical evolution of the disk, and claim to reconsider the models thus far for the disk including the solar neighborhood, in which the metallicity is predicted to monotonously increase with time. Furthermore, we anticipate that a flattening of abundance gradient together with a metal-rich floor in the outer disk are the hallmark of disk galaxies with significant central bulges.
\end{abstract}

Keywords. Galaxy: bulge - Galaxy: disk - Galaxy: evolution - ISM: jets and outflows

\section{Introduction}

How do disk galaxies form and how do they evolve through cosmic time? Clear answers to these questions continue to elude us, and it may be many years before we converge on a successful physical model. Disks lie at the forefront of galaxy formation and evolution, not least because most stars are in disks today (Benson et al. 2007, Driver et al. 2007). It is widely recognized that N-body simulations of galaxy formation within cold dark matter (CDM) cosmology fail to produce realistic galactic disks (Navarro \& White 1994, Navarro et al. 1995, Steinmetz \& Müller 1995). But a useful aspect of these incomplete models has been to highlight the possible role of feedback in shaping galaxies. Vigorous feedback in the early phase of galaxy formation is a critical solution to the angular momentum problem by preventing baryons from loosing their specific angular momentum too much through the interaction with dark matter (Fall 2002). Several recent developments have provided the impetus for the present work. In particular, disk galaxies are now being traced over 10 or more optical scale lengths to reveal an $[\mathrm{Fe} / \mathrm{H}]$ abundance gradient that declines before it flattens off in the outer disk (Yong et al. 2006 and also see contribution by Vlajic, this volume). We believe that this abundance transition is a fossil record of long-term feedback and accretion processes, as we discuss. 
Here we consider the effects of large-scale winds in the chemical evolution of disks. Several new observations suggest outflows are important in the lifecycle of galaxies. First, Tremonti et al. (2004) find evidence for chemical enrichment trends throughout starforming galaxies over three orders of magnitude in stellar mass. Indeed, there is evidence that galaxies with the mass of the Milky Way or higher manage to retain a large fraction of their metals, in contrast to lower mass galaxies where metal loss appears to be anticorrelated with baryonic mass. Recent theoretical work has proved that a low-metal content in dwarf galaxies is attributable to efficient metal-enriched outflows (Dalcanton 2007). Secondly, there is now strong evidence for large-scale outflows in the Galaxy across the electromagnetic spectrum (Bland-Hawthorn \& Cohen 2003, Fox et al. 2005, Keeney et al. 2006, Everett et al. 2008). Finally, the intergalactic medium at all redshifts shows signs of significant metal enrichment consistent with the action of winds (Cen \& Ostriker 1999, Madau et al. 2001, Ryan-Webber et al. 2006, Davé et al. 2008).

Therefore it is important to consider the influence of large-scale winds within Galactic chemical evolution (GCE) models. The key observational constraint that we consider is the Galactic abundance gradient determined from different astrophysical sources (e.g., $\mathrm{K}$ giants, HII regions, etc.). However, it confronts the fact that the simple GCE models in which the disk is divided into independently evolving rings successfully reproduce the present abundance gradient along the disk (e.g., Boissier \& Prantzos 1999, Hou et al. 2000, Chiappini et al. 2001) and the necessity to invoke winds for the chemical evolution of the Galaxy disk has not been claimed. Thus, in fact, the connection of abundance gradient with winds has been disregarded so far. Recent finding on the time evolution of abundance gradient has imposed the new task on GCE models. The observed gradient has flattened out by half in the last 5 Gyr or so (e.g., Daflon \& Cunha 2004, Maciel et al. 2006). Such a large change in abundance gradient seems beyond the predictions by any GCE models so far, and a search for its potential mechanism will invoke some ingredient to be considered in the GCE model. Then, we first propose that the observed flattening phenomenon is attributable to the once enrichment by large-scale winds.

\section{Chemical signatures of large-scale winds}

Here we consider possible evidence for large-scale winds buried within stellar abundance data. In summary, the compelling evidence for large-scale winds we insist is that we commonly see the remarkable enrichment for some period in each local region of the disk, subsequently followed by a decrease in abundances.

\subsection{Solar neighborhood}

Detailed elemental abundances of stars and gas in the solar neighborhood indicate that the presence of metal-rich stars with $[\mathrm{Fe} / \mathrm{H}] \gtrsim 0$ is the end result of additional enrichment by large-scale winds several Gyr ago, in terms of the following three aspects.

1. stellar abundance distribution function

The abundance distribution function (ADF) of solar neighborhood disk stars has been studied by many authors thus far, and the location of its peak at $[\mathrm{Fe} / \mathrm{H}]_{\text {peak }} \sim-0.2--0.1$ has been firmly established. On the other hand, the metal-rich end of ADF extends at least to $[\mathrm{Fe} / \mathrm{H}] \sim+0.2$, and the fraction of stars with $[\mathrm{Fe} / \mathrm{H}]>0$ is roughly $20 \%$. Spectroscopic observations of elemental abundances for metal-rich disk stars (Feltzing \& Gustafsson 1998, Bensby et al. 2005) have confirmed that chemical enrichment in the solar neighborhood has continuously proceeded until $[\mathrm{Fe} / \mathrm{H}] \sim+0.4$. Tsujimoto $(2007)$ has claimed that the simultaneous reproduction of both the presence of stars with $[\mathrm{Fe} / \mathrm{H}] \gtrsim+0.2$ and $[\mathrm{Fe} / \mathrm{H}]_{\text {peak }}<0$ is hard to realize through the conventional scheme of local enrichment 

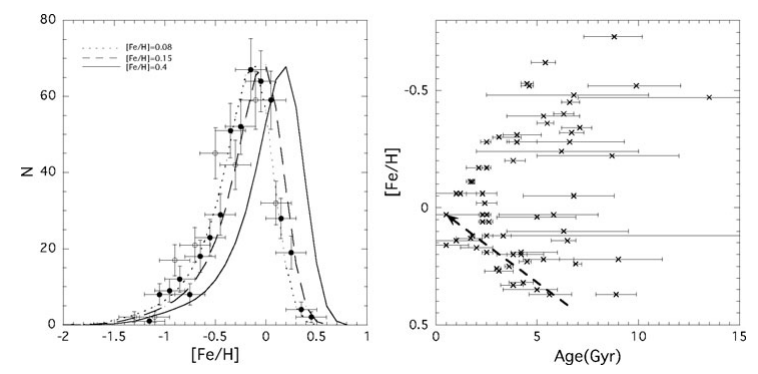

Figure 1. left panel: Abundance distribution function of disk stars against the iron abundance. Three curves are the predictions of the model with different input values of the present $[\mathrm{Fe} / \mathrm{H}]$ abundance. The calculated distributions are convolved using the Gaussian with a dispersion of 0.15 dex in $[\mathrm{Fe} / \mathrm{H}]$. Open circles and crosses represent data taken from Edvardsson et al. (1993) and Wyse \& Gilmore (1995), respectively. right panel: Age-metallicity relation of disk stars taken from Bensby et al. (2005). The trend for metal-richs stars is indicated by the dashed arrow.

under a continuous low-metal infall from the halo (see left panel of Fig. 1), and that the presence of metal-richer stars than the solar is a crucial evidence for the enrichment by outflow from the bulge.

2. offset with the present abundance

There is little evidence that the present local gas metallicity is as high as $[\mathrm{Fe} / \mathrm{H}]$ $>+0.2$. Samples of nearby young stars such as Cepheids (Lemasle et al. 2007) or OB stars (Daflon \& Cunha 2004) have a mean of around solar, including the stars in the Orion association (Cunha et al. 1998), with little or no offset with the HII region (e.g., Simon-Díaz 2006). Therefore, there exists a clear discrepancy between the presence of local metal-rich $\mathrm{F} / \mathrm{G}$ stars with $[\mathrm{Fe} / \mathrm{H}]$ up to $\sim+0.2-0.4$ and the mean metallicity of gas in the local disk as long as we take a view of the standard chemical evolution in which the metallicity increases with time. This offset should be explained by the scheme that considers a one-time, early phase rapid enrichment and a subsequent dilution.

\section{3. stellar age}

In the observed age-metalliicty relation of local disk stars (Bensby et al. 2005, Reid et al. 2007), there seems a lack of young ages for metal-rich stars, implying that these stars are not formed in the recent star formation but a few Gyr ago (see right panel of Fig. 1). This observed fact is compatible with the offset discussed above.

\subsection{Flattening}

The radial metallicity gradient roughly from $R_{\mathrm{GC}}=4$ to $R_{\mathrm{GC}}=14 \mathrm{kpc}$ has flattened out in the last several Gyr with the change in a slope of $\sim-0.1 \mathrm{dex} \mathrm{kpc}^{-1}$ to $\sim-0.04$ dex kpc ${ }^{-1}$ (e.g., Daflon \& Cunha 2004, Maciel et al. 2004). Chemical evolution models predict contradictory time evolution of metallicity gradient, i.e., a steepening (Chiappini et al. 2001) or a flattening (Hou et al. 2000). Putting aside their contradiction, these two models in common predict a monotonous increase in abundances for each region, and the predicted absolute change in gradient with time is too small as compared with the observation (see Fig. 10 of Maciel et al. 2006). Thus, it is likely that we have missed some mechanism for a flattening, and we propose that a new light on this issue is brought by a comparison of the metallicity distribution for open clusters with that for Cepheids, which tells us a hint on how the metallicity gradient flattened with time, as indicated by Figure 2; The once steep gradient became shallower owing to a decrease in metalllicity at inner regions, while an increase at outer regions.

This poses the question of whether there exists a theoretical scheme to explain such a puzzling change in abundance gradient. Our proposed idea is that large-scale winds 
from the bulge once enriched the disk. While wind entrainment is not well understood, it is now well established that winds can carry local ISM gas into the flow (Veilleux et al. 2005). It seems plausible that much of the metal-enriched gas does not travel far and falls close to the bulge. Alternatively, some of the wind explosions may not develop fully and collapse before they progress very far. In either case, metals from the enriched core can be spread over the inner disk with its abundance gradient steeper. Since the steep gradient is an outcome of the action of winds, its temporal gradient got back to a shallow one after the event of ravaging winds while each region experienced chemical enrichment under a continuous low-metal accretion from the halo.

\subsection{Outer disk}

In the outer disk, we see the abundances in giants (Yong et al. 2005, Carney et al. 2005) flatten with a metallicity of $[\mathrm{Fe} / \mathrm{H}] \sim-0.5$ in a spectacular fashion (see Fig. 16 of Yong et al. 2006). On the other hand, the abundances in Cepheids (Yong et al. 2006) does not flatten off as much, and in fact they are offset downwards in $[\mathrm{Fe} / \mathrm{H}]$ with a scatter of $-1 \lesssim[\mathrm{Fe} / \mathrm{H}] \lesssim-0.5$. According to our view, this reversed age-metallicity relation between old and young stars in the outer disk is attributable to the dilution by a low-metal infall during the last several Gyr after a setup of the metallicity floor by large-scale winds. Without the enrichment by winds, the metallicity would not reach even $[\mathrm{Fe} / \mathrm{H}] \sim$ -1 in the outer disk. Thus, this too much enrichment by winds for the outer disk would result in little reflection of self-enrichment in situ on stellar abundances until the present. This consideration gives a reasonable answer to the puzzling abundance feature of the enhanced $[\alpha$-element/Fe] ratio exhibited by all outer disk stars (Yong et al. 2006), since both large-scale winds and a low-metal infall from outside the Galaxy should exhibit a SNII like elemental feature. Yong et al. (2006) claimed that Cepheids with the metallicities lower than red giants should be the comer cannibalized in a recent merger event. However, in fact, at some region such as a so-called Cloud 2 in the outer disk $\left(R_{\mathrm{GC}} \sim 20 \mathrm{kpc}\right)$, the star formation is now ongoing (Yasui et al. 2008) in the molecular cloud with its metallicity of $[\mathrm{O} / \mathrm{H}] \sim-0.7$ (Ruffle et al. 2007), implying $[\mathrm{Fe} / \mathrm{H}] \sim-1$.

\section{Modeling of a flattening}

We try to reproduce a flattening of abundance gradient along the disk, by incorporating the enrichment by large-scale winds into the model. The basic picture is that the disk was formed through a continuous low-metal infall of material from outside the disk region based on the inside-out formation scenario (Matteucci \& François 1989), that is, the disk is formed by an infall of gas occurring at a faster rate in the inner region than in the outer ones, and for a specific period heavy elements carried by winds from the bulge dropped and enriched the disk. Here we calculate chemical evolutions at three regions with their Galactocentric distances $R=4,8$ (solar vicinity), and $12 \mathrm{kpc}$. Details of our model are described in Tsujimoto et al. (2008, in preparation).

The left panel of Figure 2 shows the predicted age-metallicity relations at three different $R$, and the corresponding $[\mathrm{Fe} / \mathrm{H}]$ ratios at $T=4 \mathrm{Gyr}$ and the present are shown as a function of $R$ on the right panel. You see that our proposed mechanism for a flattening is a decrease and an increase in abundances at inner and outer regions, respectively, after the construction of a temporally steep gradient by wind enrichment. Predicted somewhat higher $[\mathrm{Fe} / \mathrm{H}]$ abundances at a solar circle than the observed data are the results calculated with the model which is devised to be in tune with the observed chemical quantities of the solar neighborhood stars. Figure 3 shows the resultant ADF and correlation of $[\mathrm{Mg} / \mathrm{Fe}]$ with $[\mathrm{Fe} / \mathrm{H}]$, which are in good agreement with the observations. It 

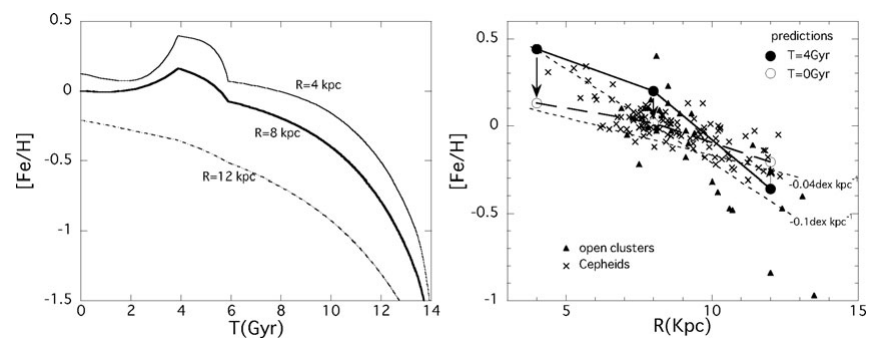

Figure 2. left panel: Predicted age- $[\mathrm{Fe} / \mathrm{H}]$ relations at the distances of $R_{\mathrm{GC}}=4,8$, and $12 \mathrm{kpc}$. The age of galaxy is assumed to be $14 \mathrm{Gyr}$. right panel: Observed $[\mathrm{Fe} / \mathrm{H}]$ distributions as a function of the distance from the center for Cepheids (crosses; Andrievsky et al. 2004) and open clusters (filled triangles; Friel 2006), compared with the predicted values at $T=4$ Gyr (filled circles) and the present (open circles), which are extracted from the results of the left panel. The $[\mathrm{Fe} / \mathrm{H}]$ ratios for Cepheids are shifted by -0.16 dex as performed by Yong et al. (2006). The directions of change in $[\mathrm{Fe} / \mathrm{H}]$ from $T=4$ Gyr to $T=0$ are denoted by arrows for each region. The observationally implied $[\mathrm{Fe} / \mathrm{H}]$ gradients are assigned by dotted lines for the present $\left(-0.04 \mathrm{dex} \mathrm{kpc}^{-1}\right)$ and several Gyr ago $\left(-0.1 \mathrm{dex} \mathrm{kpc}^{-1}\right)$.

should be stressed that the upturning feature of $[\mathrm{Mg} / \mathrm{Fe}]$ for $[\mathrm{Fe} / \mathrm{H}] \gtrsim 0$ strongly implies the enrichment by large-scale winds, and the subsequent decrease in abundances well explains an offset of abundances between the present gas and metal-rich stars. Furthermore, the origin of metal-rich stars in the solar neighborhood cannot be attributable to contaminants coming from the inner disk or the Galactic bulge as a result of radial mixing (Sellwood \& Binney 2002), since their elemental features represented by an upturning feature are at odds with those exhibited by the stars orbiting near the Galactic center.
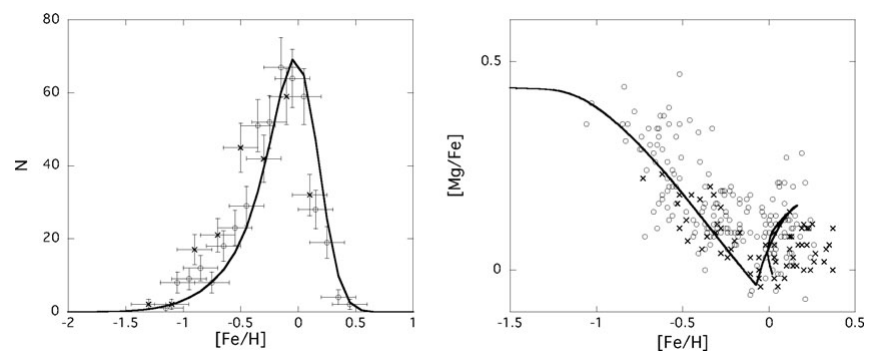

Figure 3. Features of chemical evolution in the solar neighborhood. left panel: Predicted abundance distribution function of thin disk stars against the iron abundance. right panel: Correlation of $[\mathrm{Mg} / \mathrm{Fe}]$ with $[\mathrm{Fe} / \mathrm{H}]$ for thin disk stars. The open circles and crosses are taken from Edvardsson et al. (1993) and Bensby et al. (2005), respectively.

\section{Conclusions}

We claim that large-scale winds are the crucial factor for the chemical evolution of disks. Our proposed scenario is (i) winds once set up a steep abundance gradient and a metal-rich floor in the outer disk owing to heavy elements entrained in the winds, and (ii) later evolution leads to a flattening of abundance gradient through chemical enrichment with a low-metal infall from the halo. Accordingly, we predict that a flattening and metalrich floor are the hallmark of disk galaxies with significant central bulges, and thereby the variation in wind signature among galaxies imprinted on the disk should be correlated with the size of their bulges. Our scenario poses the challenging issues which will be verified by future work. One is about the veiled star formation history of the Galactic 
bulge. We believe that the bulge has a complex history involving at least one major starburst episode around 5 Gyr ago in addition to the initial burst, and hopefully it will be unveiled with an infrared eye by the Japanese future astrometric satellite JASMINE (Gouda et al. 2008). Another issue is on the question of whether and how the chemical evolution of the Galactic disk inside a solar circle during the last Gyr has deviated from the standard picture predicted by GCE models.

\section{References}

Andrievsky, S. M., Luck, R. E., Martin, P., \& Lépine, J.R.D. 2004, A\&A, 413, 159

Bensby, T., Feltzing, S., Lundström, I., \& Ilyiin, I. 2005, A\&\&A, 433, 185

Benson, A. J., Džanović, D., Frenk, C. S., \& Sharples, R. 2007, MNRAS, 379, 841

Sellwood, J. A. \& Binney, J. J. 2002, MNRAS, 336, 785

Bland-Hawthorn, J. \& Cohen, M. 2003, ApJ, 582, 246

Boissier, S. \& Prantzos, N. 1999, MNRAS, 307, 857

Carney, B. W., Yong, D., de Almeida, L., \& Seitzer, P. 2005, AJ, 130, 1111

Cen, R. \& Ostriker, J. P. 1999, ApJ, 514, 1

Chiappini, C., Matteucci, F., \& Romano, D. 2001, ApJ, 554, 1044

Cunha, K., Smith, V. V., \& Lambert, D. L. 1998, ApJ, 493, 195

Daflon, S. \& Cunha, K. 2004, ApJ, 617, 1115

Dalcanton, J. J. 2007, ApJ, 658, 941

Davé, R., Oppenheimer, B. D., \& Sivanandam, S. 2008, submitted to MNRAS

Driver, S. P., Allen, P. D., Liske, J., \& Graham, A. W. 2007, ApJ, 657, L85

Edvardsson, B. et al. 1993, A\&SA, 275, 101

Everett, J. E. et al. 2008, ApJ, 674, 258

Fall, S. M. 2006, in: E. Athanassoula, A. Bosma, \& R. Mujica (eds.) Disks of Galaxies: Kinematics, Dynamics and Perturbations (San Francisco: ASP), p. 389

Feltzing, S. \& Gustafsson, B. 1998, A\&AS, 129, 237

Friel, E. D. 2006, in: L. Pasquini \& S. Randich (eds.) Chemical Abundances and Mixing in Stars in the Milky Way and its Satellites (Berlin: Springer), p. 3

Fox, A. J. et al. 2005, ApJ, 630, 332

Gouda, N. et al. 2008, in: W.-J. Jin, I. Platais, \& M. Perryman (eds.), A Giant Step: from Millito Micro-arcsecond Astrometry, Proc. IAU Symp. No. 248 (San Francisco: ASP), p. 248

Hou, J. L., Prantzos, N., \& Boissier, S. 2000, A\&A, 362, 921

Keeney, B. A. et al. 2006, ApJ, 646, 951

Lemasle, B. et al. 2007, A\&BA, 467, 283

Maciel, W. L., Lago, L. G., \& Costa, R. D. D. 2006, A\&A, 453, 587

Madau, P., Ferrara, A., \& Rees, M.J. 2001, ApJ, 555, 92

Matteucci, F. \& François, P. 1989, MNRAS, 239, 885

Navarro, J. F. \& White, S. D. M. 1994, MNRAS, 267, 401

Navarro, J. F., Frenk, C. S., \& White, S. D. M. 1995, MNRAS, 275, 56

Reid, I. N., Turner, E. L., Turnbull, M. C., Mountain, M., \& Valenti, J. A. 2007, ApJ, 665, 767

Ruffle, P. M. E. et al. 2007, ApJ, 671, 1766

Ryan-Weber, E. V., Pettini, M., \& Madau, P. 2006, MNRAS, 371, L78

Simón-Díaz, S. 2006, astro-ph/0611513

Steinmetz, M. \& Müller, E. 1995, MNRAS, 276, 549

Tremonti, C. A. et al. 2004, ApJ, 613, 898

Tsujimoto, T. 2007, ApJ, 665, L115

Veilleux, S., Cecil, G., \& Bland-Hawthorn, J. 2005, ARAESA, 43, 769

Wyse, R. F. G. \& Gilmore, G. 1995, AJ, 110, 2771

Yasui, C., Kobayashi, N., Tokunaga, A. T., Terada, H., \& Saito, M. 2008, ApJ, 675, 443

Yong, D., Carney, B. W., \& de Almeida, L. 2005, AJ, 130, 597

Yong, D., Carney, B. W., Luísa, M., de Almeida, L., \& Pohl, B. L. 2006, AJ, 131, 2256 